\title{
On Size-Biased Double Weighted Exponential Distribution (SDWED)
}

\author{
Zahida Perveen $^{1 *}$, Zulfiqar Ahmed ${ }^{2}$, Munir Ahmad ${ }^{3}$ \\ ${ }^{1}$ Lahore Garrison University, Main Campus Sector C, Phase VI, DHA Lahore, Pakistan \\ ${ }^{2}$ GIFT University, Gujranwala, Pakistan \\ ${ }^{3}$ National College of Business Administration and Economics, Lahore, Pakistan \\ Email: ^drzahida95@gmail.com,dr.xxee@gmail.com
}

How to cite this paper: Perveen, Z., Ahmed, Z. and Ahmad, M. (2016) On Size-Biased Double Weighted Exponential Distribution (SDWED). Open Journal of Statistics, 6, 917930.

http://dx.doi.org/10.4236/ojs.2016.65076

Received: July 25, 2016

Accepted: October 24, 2016

Published: October 27, 2016

Copyright (c) 2016 by authors and Scientific Research Publishing Inc. This work is licensed under the Creative Commons Attribution International License (CC BY 4.0).

http://creativecommons.org/licenses/by/4.0/ (c) (i) Open Access

\begin{abstract}
This paper introduces a new distribution based on the exponential distribution, known as Size-biased Double Weighted Exponential Distribution (SDWED). Some characteristics of the new distribution are obtained. Plots for the cumulative distribution function, pdf and hazard function, tables with values of skewness and kurtosis are provided. As a motivation, the statistical application of the results to a problem of ball bearing data has been provided. It is observed that the new distribution is skewed to the right and bears most of the properties of skewed distribution. It is found that our newly proposed distribution fits better than size-biased Rayleigh and Maxwell distributions and many other distributions. Since many researchers have studied the procedure of the weighted distributions in the estates of forest, biomedicine and biostatistics etc., we hope in numerous fields of theoretical and applied sciences, the findings of this paper will be useful for the practitioners.
\end{abstract}

\section{Keywords}

Exponential Distribution, Moments, Moment Ratios, Estimation

\section{Introduction}

Weighted distributions are suitable in the situation of unequal probability sampling, such as actuarial sciences, ecology, biomedicine biostatistics and survival data analysis. These distributions are applicable, when observations are recorded without any experiment, repetition and random process. The notion of weighted distributions has been used as a device for the collection of suitable model for observed data, during last 25 years. The idea is most applicable when sampling frame is not available and random sampling is not possible. Firstly the idea of weighted distributions was introduced by 
Fisher [1]. Cox [2] firstly provided the idea of length-biased sampling and after that Rao [3] established a unifying method that can be used for several sampling situations and can be displayed by means of the weighted distributions. Cox [4] estimated the mean of the original distribution built on length-biased data. Zelen [5] presented the concept of weighted distribution in studying cell kinetics and early discovery of disease. Warren [6] applied these distributions in forest product research. Patil and Rao [7] surveyed the idea and applications of weighted and size-biased sampling distributions. Patil and Rao [8] also discussed weighted binomial distribution to model the human families and estimation of the wildlife family size. Gupta and Keating [9] described the relationship between reliability measures of original and size-biased distribution. Arnold and Nagaraja [10] gave the idea of bivariate weighted distribution whereas Jain and Nanda [11] extended this idea and discussed multivariate aspect of weighted distribution.

Let $f(x ; \theta)$ be the pdf of the random variable $x$ and $\theta$ be the unknown parameter.

The weighted distribution is defined as;

$$
g(x ; \theta)=\frac{w(x) f(x ; \theta)}{E[w(x)]}, \quad x \in R, \theta>0
$$

where $w(x)$ is a weight function. When $w(x)=x^{m}$, then these distributions are termed as size-biased distribution of order $m$. When $m=1$ it is called size-biased of order 1 or say length biased distribution, whereas for $m=2$ it is called the area-biased distribution (Ord and Patil [12], Patil [13] and Mahfound [14]).

In forest product research, equilibrium and length biased distributions have been used as moment distributions. Kochar and Gupta [15] discussed the moment distributional properties in assessment with the actual distributions and derived the bound on the moments of moment distributions.

Oluyede [16] described inequalities for the reliability measures of size-biased and the original distributions. Navarro et al. [17] discussed characterization of the original and the size-biased distribution using reliability measures. Gove [18] offered the uses of size-biased distributions in forest science and ecology. Sunoj and Maya [19] established relationships among weighted and original distributions in the situation of repairable system and also characterized the sized-biased and the original distribution. Shen et al. [20] used semi-parametric transformations to model the length biased data. Hussain and Ahmad [21] presented misclassification in the size-biased modified power series distributions and its applications.

Mir and Ahmad [22] derived generalized forms of size-biased discrete distributions and discussed the practical applications in the field of Medical, Zoology and Accidental studies. Mir [23] derived size-biased Geeta distribution and size-biased consul distribution respectively, different properties are discussed and contrasts with original distributions are also done. Das and Roy [24] established size-biased form of generalized Rayleigh distribution and apply the consequences to the environmental data. They also applied the concept of size-biased sampling in the field of environmental studies

Dara [25] derived reliability measures for size-biased forms of several moment distributions as the special cases of moment distributions. Iqbal and Ahmad [26] found 
compound scale mixtures of limiting distribution of generalized log Pearson type VII distribution with different continuous and moment distributions. Hasnain [27] introduced a new family of distributions named as exponentiated moment exponential (EME) distribution and developed its properties. Iqbal et al. [28] found a more general class for EME distribution and built up different properties including characterization through conditional moments.

Zahida and Munir [29] worked on Weighted Weibull Distributions (WWD), Double Weibull Distributions (DWD), Weighted Double Weibull Distributions (WDWD), Double Weighted Exponential Distributions (DWED) (both in size-biased and area biased). Some basic theoretical properties of all these distributions including cumulative density function, central moments, skewness, kurtosis and moments are studied. Shannon entropy, Renyi entropy, moment generating function and information generating function of all these distributions are derived. Reliability measures including survival function, failure rates, reverse hazard rate function and Mills ratios of these distributions are also obtained. Parameters are evaluated by using method of maximum likelihood estimation along with derivation of practical examples.

The exponential distribution has a fundamental role in describing a large class of phenomena, particularly in the area of reliability theory. This distribution is commonly used to model waiting times between occurrences of rare events, lifetimes of electrical or mechanical devices. It is also used to get approximate solutions to difficult distribution problems.

\section{Methodology}

\subsection{Size-Biased Double Weighted Exponential Distribution (SDWED)}

The size-biased double weighted exponential distribution is given by:

$$
g_{0}(x ; \lambda, c)=\frac{f(x) F(c x)}{\int_{0}^{\infty} f(x) F(c x) \mathrm{d} x}, x \geq 0, \lambda, c>0
$$

where $f(x)$ is the first weight and $f(x)=\frac{w(x) g(x)}{\int_{0}^{\infty} x g(x) \mathrm{d} x}$

Here $w(x)=x$ and $g(x)=\lambda \mathrm{e}^{-\lambda x}, \lambda>0, x \geq 0$ is the pdf of exponential distribution.

Thus the pdf of SDWED is

$$
g_{0}(x ; \lambda, c)=\frac{\lambda^{2}(1+c)^{3}}{c^{3}+3 c^{2}}\left[x \mathrm{e}^{-\lambda x}\left(1-\mathrm{e}^{-\lambda c x}-\lambda c x \mathrm{e}^{-\lambda c x}\right)\right], x \geq 0, \lambda, c>0
$$

where $\lambda$ is shape parameter and $c$ is scale parameter.

Graphs of Probability Density Function

Figure 1 and Figure 2 show the probability density function of SDWED.

\subsection{Distribution Function of SDWED}

Distribution function of a density function is defined as: 


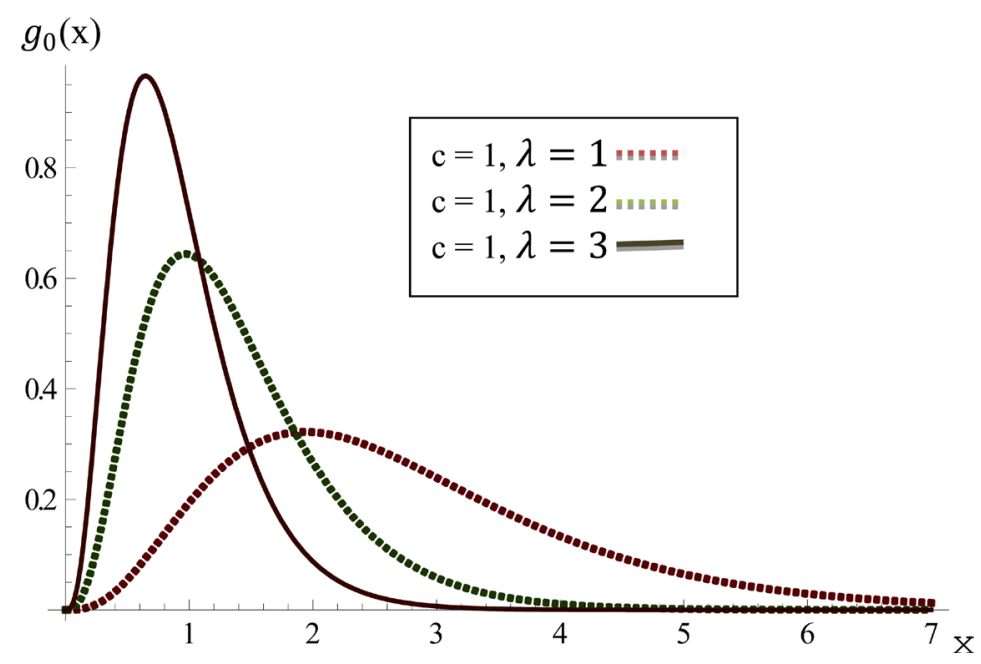

Figure 1. The probability density function of SDWED for the indicated values of $c$ and $\lambda$.

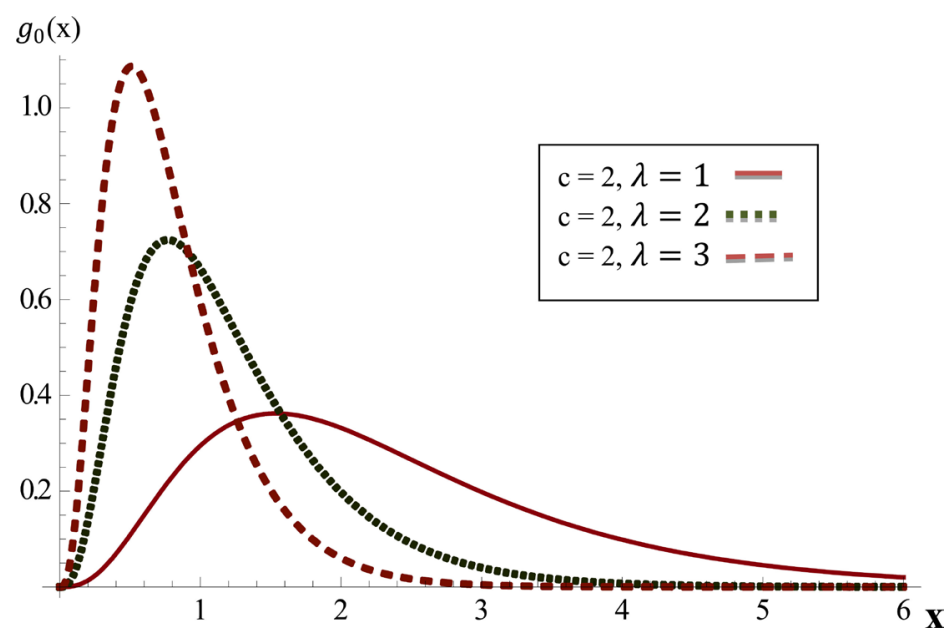

Figure 2. The probability density function of SDWED for the indicated values of $c$ and $\lambda$.

$$
\begin{gathered}
F(x ; \lambda, c)=\int_{0}^{x} h(t) \mathrm{d} t \\
F(x)=\frac{\lambda^{2}(1+c)^{2}}{c^{3}+3 c^{2}} \int_{0}^{x} t \mathrm{e}^{-\lambda t}\left(1-\mathrm{e}^{-\lambda t}-\lambda c t \mathrm{e}^{-\lambda c t}\right) \mathrm{d} t
\end{gathered}
$$

After some simplification we have:

$$
\begin{aligned}
F(x)= & \frac{(1+c)^{3}\left[1-\mathrm{e}^{-\lambda x}-\lambda x \mathrm{e}^{-\lambda x}\right]}{c^{3}+3 c^{2}}-\frac{(1+c)\left[1-\mathrm{e}^{-\lambda x(1+c)}-\lambda x(1+c) \mathrm{e}^{-\lambda x(1+c)}\right]}{c^{3}+3 c^{2}} \\
& -\frac{\left[-\lambda^{2}(1+c)^{2}\left(x^{2} \mathrm{e}^{-\lambda x(1+c)}\right)-2 \lambda x(1+c) \mathrm{e}^{-\lambda x(1+c)}-2 \mathrm{e}^{-\lambda x(1+c)}+2\right]}{c^{2}+3 c}
\end{aligned}
$$

Figure 3 shows the commutative distribution function of SDWED.

\subsection{Survival Function}

The survival function of SDWED is defined as 


$$
\begin{aligned}
S(x)= & -F(x) \\
S(x)= & 1-\frac{(1+c)^{3}\left[1-\mathrm{e}^{-\lambda x}-\lambda x \mathrm{e}^{-\lambda x}\right]}{c^{3}+3 c^{2}}-\frac{(1+c)\left[1-\mathrm{e}^{-\lambda x(1+c)}-\lambda x(1+c) \mathrm{e}^{-\lambda x(1+c)}\right]}{c^{3}+3 c^{2}} \\
& -\frac{\left[-\lambda^{2}(1+c)^{2}\left(x^{2} \mathrm{e}^{-\lambda x(1+c)}\right)-2 \lambda x(1+c) \mathrm{e}^{-\lambda x(1+c)}-2 \mathrm{e}^{-\lambda x(1+c)}+2\right]}{c^{2}+3 c}
\end{aligned}
$$

Figure 4 shows the survival function of SDWED.

\subsection{Hazard Rate Function of SDWED}

The hazard rate function is defined as:

$$
h(x)=\frac{g_{0}(x)}{S(x)}
$$

At $c=1$ and $\lambda=1$, the hazard rate function will be:

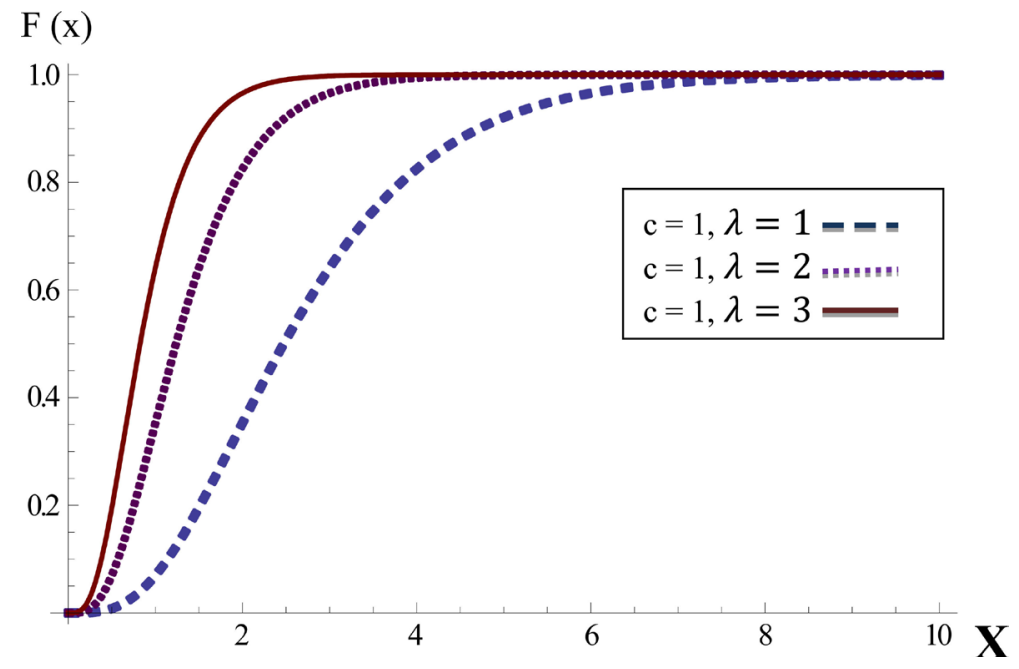

Figure 3. Cumulative Distribution Function of SDWED for the indicated values of $c$ and $\lambda$.

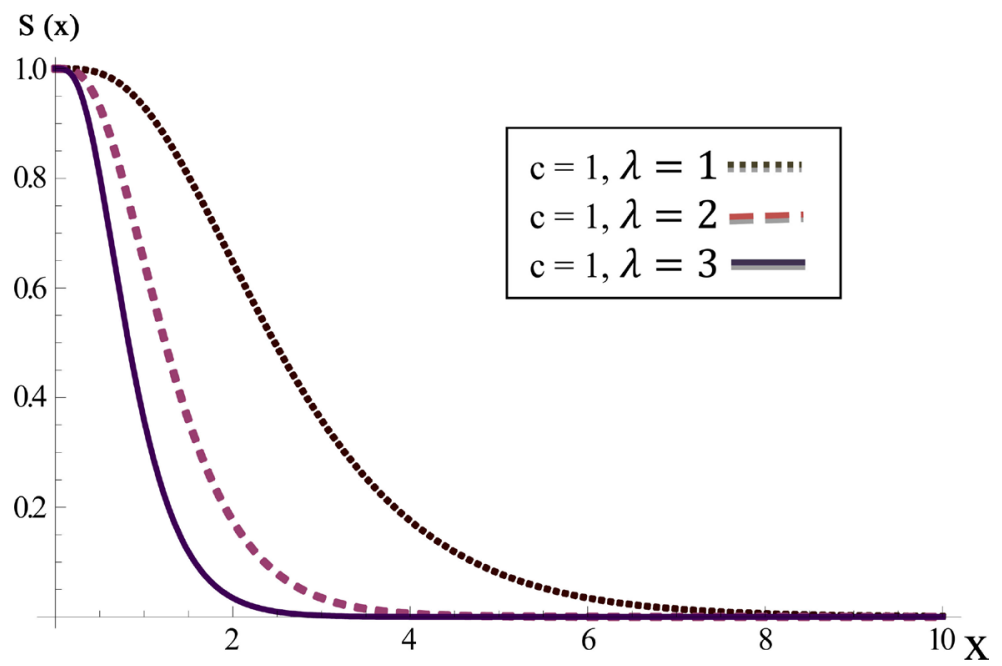

Figure 4. The Survival Function of SDWED for the indicated values of $c$ and $\lambda$. 


$$
h(x)=\frac{2 x \mathrm{e}^{-x}-2 x \mathrm{e}^{-2 x}-2 x^{2} \mathrm{e}^{-2 x}}{1-2 \mathrm{e}^{-x}-2 x \mathrm{e}^{-2 x}+\mathrm{e}^{-2 x}+1.25 x \mathrm{e}^{-2 x}+x^{2} \mathrm{e}^{-2 x}}
$$

Figure 5 shows the Hazard Rate Function of SDWED.

\subsection{Reverse Hazard Rate Function}

The reverse Hazard rate function of SDWED is given by

$$
r(x)=\frac{g_{0}(x)}{F(x)}
$$

At $c=1$ and $\lambda=1$, the reverse hazard rate function will be:

$$
r(x)=\frac{2 x \mathrm{e}^{-x}-2 x \mathrm{e}^{-2 x}-2 x^{2} \mathrm{e}^{-2 x}}{2 \mathrm{e}^{-x}+2 x \mathrm{e}^{-2 x}-\mathrm{e}^{-2 x}-1.25 x \mathrm{e}^{-2 x}-x^{2} \mathrm{e}^{-2 x}}
$$

Figure 6 shows the Reverse Hazard Rate Function of SDWED.

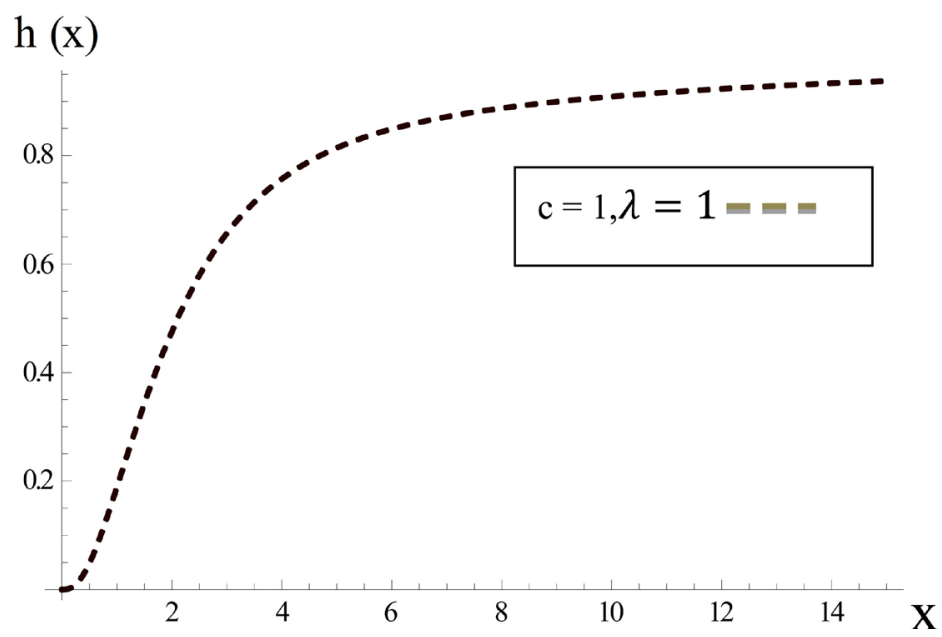

Figure 5. The Hazard Function of SDWED for the indicated values of $\mathrm{c}$ and $\lambda$.

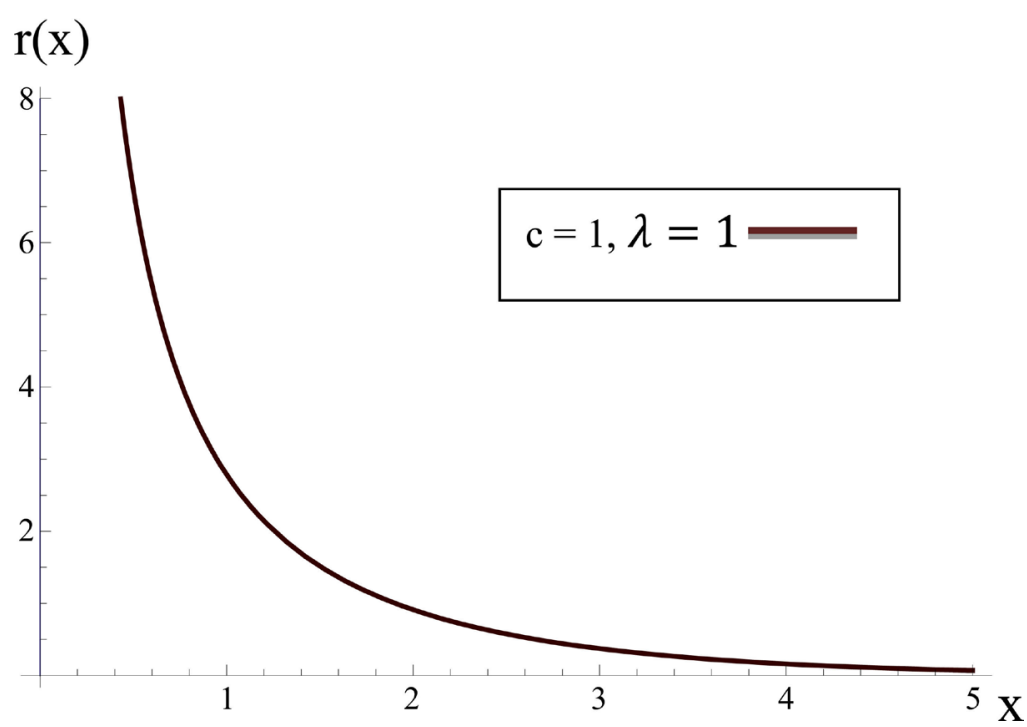

Figure 6. Reverse Hazard Function of SDWED for the indicated values of $c$ and $\lambda$. 


\subsection{Mills Ratio}

The Mills Ratio is given by:

$$
m(x)=\frac{1}{h(x)}
$$

At $c=1$ and $\lambda=1$, the Mills Ratio will be:

$$
m(x)=\frac{1-2 \mathrm{e}^{-x}-2 x \mathrm{e}^{-2 x}+\mathrm{e}^{-2 x}+1.25 x \mathrm{e}^{-2 x}+x^{2} \mathrm{e}^{-2 x}}{2 x \mathrm{e}^{-x}-2 x \mathrm{e}^{-2 x}-2 x^{2} \mathrm{e}^{-2 x}}
$$

Figure 7 shows the mills ratio of SDWED.

\subsection{Moment Generating Function of SDWED}

The moment generating function of SDWED is:

$$
M_{X}(t)=\int_{0}^{\infty} \mathrm{e}^{t x} g_{0}(x) \mathrm{d} x
$$

Using Equation (3)

$$
\begin{aligned}
M_{X}(t) & =\int_{0}^{\infty} \mathrm{e}^{t x} \cdot \frac{\lambda^{2}(1+c)^{3}}{c^{3}+3 c^{2}}\left[x \mathrm{e}^{-\lambda x}\left(1-\mathrm{e}^{-\lambda c x}-\lambda c x \mathrm{e}^{-\lambda c x}\right)\right] \mathrm{d} x \\
& =\frac{\lambda^{2}(1+c)^{3}}{c^{3}+3 c^{2}} \int_{0}^{\infty}\left[x \mathrm{e}^{-x(\lambda-t)}-x \mathrm{e}^{-x(\lambda(1+c)-t)}-x^{2} \mathrm{e}^{-x(\lambda(1+c)-t)}\right] \mathrm{d} x
\end{aligned}
$$

Applying the transformations and after simplifying:

$$
M_{X}(t)=\frac{\lambda^{2}(1+c)^{3}\left[\left[(\lambda(1+c)-t)^{3}\right]-(\lambda-t)^{2}[\lambda(1+c)-t]-2 \lambda(\lambda-t)^{2} c\right]}{[\lambda(1+c)-t]^{3}\left(c^{3}+3 c^{2}\right)(\lambda-t)^{2}}
$$

\subsection{Information Generating Function of SDWED}

The information generating function is defined as:

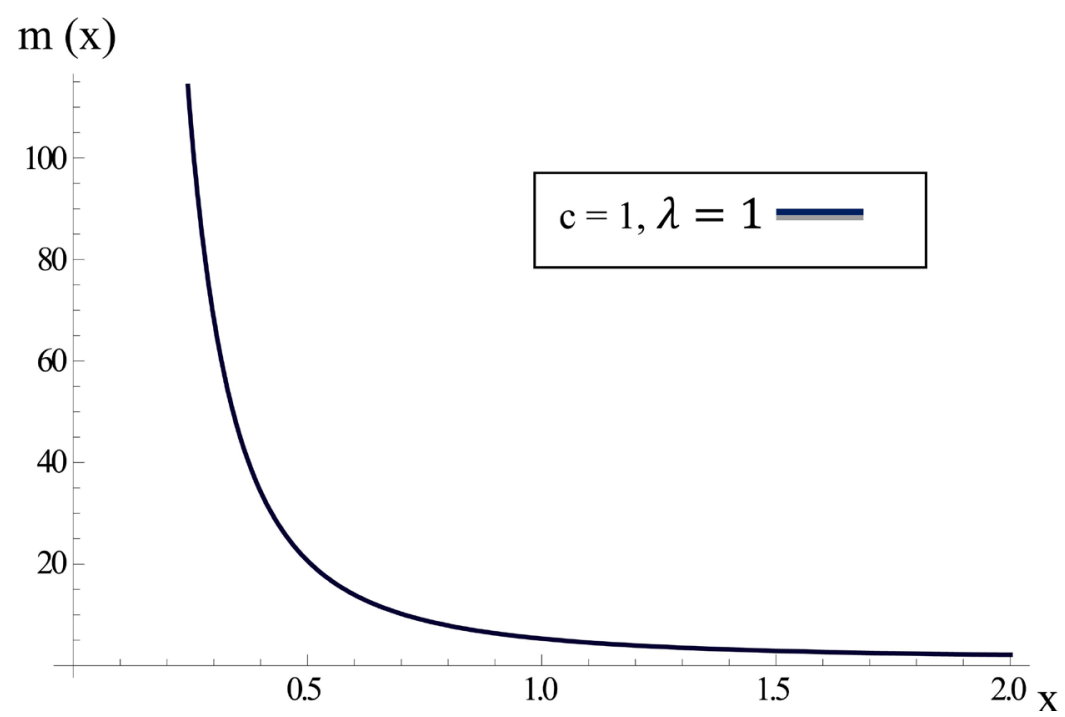

Figure 7. Mills ratio of SDWED for the indicated values of $c$ and $\lambda$. 


$$
T(s)=E\left[g_{0}(x)\right]^{s}=\int_{0}^{\infty}\left(g_{0}(x)\right)^{s} g_{0}(x) \mathrm{d} x
$$

Using Equation (3)

$$
=\frac{\lambda^{2(s+1)}(1+c)^{3(s+1)}}{\left(c^{3}+3 c^{2}\right)^{s+1}} \int_{0}^{\infty} x^{s+1} \mathrm{e}^{-\lambda x(1+s)}\left(1-\mathrm{e}^{-\lambda x c}-\lambda x c \mathrm{e}^{-\lambda x c}\right)^{1+s} \mathrm{~d} x
$$

Putting

$$
\left(1-\mathrm{e}^{-\lambda x c}-\lambda x c \mathrm{e}^{-\lambda x c}\right)^{1+s}=\sum_{i=0}^{s+1}(s+1)_{i}(-1)^{i}\left[\left((1+\lambda x c) \mathrm{e}^{-\lambda x c}\right)^{i}\right]
$$

and after a long simplification, the information generating function will be:

$$
T(s)=\sum_{i=0}^{s+1}(s+1)_{i}(-1)^{i} \frac{\lambda^{2 s}(1+c)^{3(s+1)}}{\lambda^{s+1}[c i+s+1]^{s+2}} \sum_{j=0}^{i}(i)_{j}\left(\frac{c}{c i+s+1}\right)^{j} \Gamma(s+j+2)
$$

\subsection{Limit and Mode of SDWED}

Note that the limit of the density function given in Equation (3) is as follows:

$$
\begin{aligned}
& x \rightarrow 0, g_{0}(x ; \lambda, c)=x \rightarrow 0, \frac{\lambda^{2}(1+c)^{3}}{c^{3}+3 c^{2}}\left[x \mathrm{e}^{-\lambda x}\left(1-\mathrm{e}^{-\lambda x}-\lambda c x \mathrm{e}^{-\lambda c x}\right)\right]=0 \\
& x \rightarrow \infty, g_{0}(x ; \lambda, c)=\frac{\lambda^{2}(1+c)^{3}}{c^{3}+3 c^{2}} x \rightarrow \infty, x \mathrm{e}^{-\lambda x}\left(1-\mathrm{e}^{-\lambda x}-\lambda c x \mathrm{e}^{-\lambda c x}\right)=0
\end{aligned}
$$

since

$$
x \rightarrow \infty, \mathrm{e}^{-\lambda x}=0 \text { and } x \rightarrow \infty,\left(1-\mathrm{e}^{-\lambda x}-\lambda c x \mathrm{e}^{-\lambda c x}\right)=1
$$

\subsection{Mode of SDWED}

Taking log of Equation (3) on both sides:

$$
\log \left(g_{0}(x ; \lambda, c)\right)=\log \left(\frac{\lambda^{2}(1+c)^{3}}{c^{3}+3 c^{2}}\right)+\log x-\lambda x+\log \left(1-\mathrm{e}^{-\lambda x}-\lambda c x \mathrm{e}^{-\lambda c x}\right)
$$

Differentiating Equation (15) with respect to $x$, we obtain:

$$
\frac{\partial}{\partial x} \log \left(g_{0}(x ; \lambda, c)\right)=\frac{1}{x}-\lambda+\frac{c^{2} \lambda^{2} x \mathrm{e}^{-\lambda c x}}{1-\mathrm{e}^{-\lambda c x}-\lambda c x \mathrm{e}^{-\lambda c x}}
$$

The mode of the SDWED is obtained by solving the nonlinear equation with respect to $x$.

$$
\frac{1}{x}-\lambda+\frac{c^{2} \lambda^{2} x \mathrm{e}^{-\lambda c x}}{1-\mathrm{e}^{-\lambda c x}-\lambda c x \mathrm{e}^{-\lambda c x}}=0
$$

The mode of SDWED is given in Table 1.

\subsection{Mean of SDWED}

$$
\mu(x)=\frac{2 c^{2}+8 c+12}{\lambda\left(c^{2}+4 c+3\right)}
$$


Table 1. Mode of SDWED.

\begin{tabular}{lll}
\hline$c$ & $\lambda$ & mode \\
\hline 2 & 1 & 1.543 \\
2 & 2 & 0.578 \\
2 & 3 & 0.336 \\
2 & 4 & 0.250 \\
2 & 5 & 0.200 \\
\hline
\end{tabular}

Table 2. Mean, variance and standard deviation of SDWED.

\begin{tabular}{ccccc}
\hline$c$ & $\lambda$ & Mean & Variance & Standard Deviation \\
\hline 2 & 1 & 2.4 & 1.98 & 1.41 \\
2 & 2 & 1.2 & 0.50 & 0.70 \\
2 & 3 & 0.8 & 0.04 & 0.22 \\
2 & 4 & 0.6 & 0.01 & 0.12 \\
2 & 5 & 0.4 & 0.01 & 0.08 \\
\hline
\end{tabular}

\subsection{Variance of SDWED}

$$
\sigma^{2}(x)=\frac{3 c^{7}+26 c^{6}+132 c^{5}+356 c^{4}+590 c^{3}+630 c^{2}+396 c+108}{\lambda^{2}\left(c^{7}+13 c^{6}+69 c^{5}+193 c^{4}+307 c^{3}+279 c^{2}+135 c+27\right)}
$$

Table 2 shows the Mean, Variance and Standard Deviation with some values of the parameters $\lambda$ and $\mathrm{c}$.

\subsection{Moments of SDWED}

The $r^{\text {th }}$ moment of SDWED is given by

$$
\mu_{r}^{\prime}=E\left(x^{r}\right)=\frac{\lambda^{-r}}{c^{3}+3 c^{2}}\left[(1+c)^{3} \Gamma(r+2)-(1+c)^{1-r} \Gamma(r+2)-c(1+c)^{-r} \Gamma(r+3)\right]
$$

for $r=1,2,3,4$, the first four moments about the mean are

$$
\begin{aligned}
\mu_{1}= & 0 \\
\mu_{2}= & \left(\frac{6 c^{3}+30 c^{2}+60 c+60}{\lambda^{2}\left(c^{3}+5 c^{2}+7 c+3\right)}\right)-\left(\frac{2 c^{2}+8 c+12}{\lambda\left(c^{2}+4 c+3\right)}\right)^{2} \\
\mu_{3}= & \frac{24 c^{4}+144 c^{3}+360 c^{2}+480 c+360}{\lambda^{3}\left(c^{4}+6 c^{3}+12 c^{2}+4 c+3\right)} \\
& -3\left(\frac{2 c^{2}+8 c+12}{\lambda\left(c^{2}+4 c+3\right)}\right)\left(\frac{6 c^{3}+30 c^{2}+60 c+60}{\lambda^{2}\left(c^{3}+5 c^{2}+7 c+3\right)}\right) \\
& +2\left(\frac{2 c^{2}+8 c+12}{\lambda\left(c^{2}+4 c+3\right)}\right)^{3}
\end{aligned}
$$




$$
\begin{aligned}
\mu_{4}= & \left(\frac{120 c^{5}+840 c^{4}+2520 c^{3}+4200 c^{2}+4200 c+2520}{\lambda^{4}\left(c^{5}+7 c^{4}+18 c^{3}+22 c^{2}+13 c+3\right)}\right) \\
& -4\left(\frac{2 c^{2}+8 c+12}{\lambda\left(c^{2}+4 c+3\right)}\right)\left(\frac{24 c^{4}+144 c^{3}+360 c^{2}+480 c+360}{\lambda^{3}\left(c^{4}+6 c^{3}+12 c^{2}+4 c+3\right)}\right) \\
& +6\left(\frac{2 c^{2}+8 c+12}{\lambda\left(c^{2}+4 c+3\right)}\right)^{2}\left(\frac{6 c^{3}+30 c^{2}+60 c+60}{\lambda^{2}\left(c^{3}+5 c^{2}+7 c+3\right)}\right)-3\left(\frac{2 c^{2}+8 c+12}{\lambda\left(c^{2}+4 c+3\right)}\right)^{4}
\end{aligned}
$$

\subsection{Moment Ratios}

Table 3 shows the coefficients of skewness and kurtosis.

\section{Maximum Likelihood Estimation}

The maximum likelihood estimation of SDWED distribution may be defined as:

$$
L\left(\lambda, c ; x_{1}, x_{2}, \cdots, x_{n}\right)=\prod_{i=1}^{n} g_{0}\left(x_{i}\right) .
$$

Here the independent observations are $x_{1}, x_{2}, \cdots, x_{n}$, then the likelihood function of the DWED is:

$$
\begin{gathered}
L\left(\lambda, c ; x_{1}, x_{2}, \cdots, x_{n}\right)=\sum_{i=1}^{n} \log \left(g_{0}\left(x_{i} ; \lambda, c\right)\right) \\
L(\lambda, c)=2 n \log \lambda+3 n \log (1+c)-9 n \log (c)+\sum_{i=1}^{n} \log x_{i} \\
-\lambda \sum_{i=1}^{n} x_{i}+\sum_{i=1}^{n} \log \left(1-\mathrm{e}^{-\lambda c x_{i}}-\lambda c x \mathrm{e}^{-\lambda c x_{i}}\right)
\end{gathered}
$$

This admits the partial derivatives:

$$
\frac{\partial L(\lambda, c)}{\partial \lambda}=\frac{2 n}{\lambda}-\sum_{i=1}^{n} x_{i}+\frac{n c^{2} x_{i}^{2} \lambda \mathrm{e}^{-\lambda c x_{i}}}{1-\mathrm{e}^{-\lambda c x_{i}}-\lambda c x_{i} \mathrm{e}^{-\lambda c x_{i}}}
$$

and

$$
\frac{\partial L(\lambda, c)}{\partial c}=\frac{3 n}{1+c}-\frac{9 n}{c}+\frac{n \lambda^{2} x_{i}^{2} c \mathrm{e}^{-\lambda c x_{i}}}{1-\mathrm{e}^{-\lambda c x_{i}}-\lambda c x_{i} \mathrm{e}^{-\lambda c x_{i}}}
$$

Equating these equations to zero, then we get:

$$
\frac{2 n}{\lambda}-\sum_{i=1}^{n} x_{i}+\frac{n c^{2} x_{i}^{2} \lambda \mathrm{e}^{-\lambda c x_{i}}}{1-\mathrm{e}^{-\lambda c x_{i}}-\lambda c x_{i} \mathrm{e}^{-\lambda c x_{i}}}=0
$$

Table 3. Coefficients of skewness and kurtosis of SDWED.

\begin{tabular}{cccc}
\hline$c$ & $\lambda$ & $\sqrt{\beta_{1}}$ & $\beta_{2}$ \\
\hline 2.481 & 1 & 0.0005 & 1.067 \\
2.484 & 1 & 0.0049 & 1.076 \\
2.480 & 2 & 0.0003 & 1.066 \\
3.000 & 2 & 1.3450 & 2.778 \\
3.001 & 3 & 1.0060 & 2.999 \\
\hline
\end{tabular}




$$
\frac{3 n}{1+c}-\frac{9 n}{c}+\frac{n \lambda^{2} x_{i}^{2} c \mathrm{e}^{-\lambda c x_{i}}}{x_{i}-\lambda c x_{i} \mathrm{e}^{-\lambda c x_{i}}}=0
$$

which can be solved simultaneously for $\hat{\lambda}$ and $\hat{c}$.

The asymptotic variance-covariance matrix is the inverse of $I(\xi, k, \theta)=-E(H(x))$

$$
H(x)=\left(\begin{array}{ll}
\frac{\partial^{2}\left(\log \left(g_{0}(x ; \lambda, c)\right)\right)}{(\partial \lambda)^{2}} & \frac{\partial^{2}\left(\log \left(g_{0}(x ; \lambda, c)\right)\right)}{(\partial \lambda \partial c)} \\
\frac{\partial^{2}\left(\log \left(g_{0}(x ; \lambda, c)\right)\right)}{(\partial c \partial \lambda)} & \frac{\partial^{2}\left(\log \left(g_{0}(x ; \lambda, c)\right)\right)}{(\partial c)^{2}}
\end{array}\right)
$$

The inverse of the asymptotic covariance matrix is $I(\lambda, c)=-E(H(x))$ with

$$
\begin{aligned}
\frac{\partial^{2}\left(\log \left(g_{0}(x ; \lambda, c)\right)\right)}{(\partial \lambda)^{2}} & =\frac{-2 n}{\lambda^{2}}+\frac{n c^{2} x^{2} \mathrm{e}^{-\lambda c x}(1-c x)\left(1-\mathrm{e}^{-\lambda c x}\right)-\lambda c x\left(n c^{2} x^{2} \mathrm{e}^{-2 \lambda c x}\right)}{\left(1-\mathrm{e}^{-\lambda c x}-\lambda c x \mathrm{e}^{-\lambda c x}\right)^{2}} \\
\frac{\partial^{2}\left(\log \left(g_{0}(x ; \lambda, c)\right)\right)}{(\partial c)^{2}} & =\frac{-3 n}{(1+c)^{2}}+\frac{9 n}{c^{2}}+\frac{\lambda^{2} x^{2} \mathrm{e}^{-\lambda c x}\left(1-\lambda c x-\mathrm{e}^{-\lambda c x}\right)}{\left(1-\mathrm{e}^{-\lambda c x}-\lambda c x \mathrm{e}^{-\lambda c x}\right)^{2}} \\
\frac{\partial^{2}\left(\log \left(g_{0}(x ; \lambda, c)\right)\right)}{(\partial \lambda \partial c)} & =\frac{\partial^{2}\left(\log \left(g_{0}(x ; \lambda, c)\right)\right)}{(\partial c \partial \lambda)} \\
& =\frac{2 n c \lambda x^{2} \mathrm{e}^{-\lambda c x}\left(1-\mathrm{e}^{-\lambda c x}\right)-n c^{2} \lambda^{2} x^{3} \mathrm{e}^{-\lambda c x}\left(1+\mathrm{e}^{-\lambda c x}\right)}{\left(1-\mathrm{e}^{-\lambda c x}-\lambda c x \mathrm{e}^{-\lambda c x}\right)^{2}}
\end{aligned}
$$

\section{Numerical Example}

\section{The Ball Bearing Data Records}

See for data set published in Lawless [30]

In Table 4, the approximations of the parameters are specified. For goodness-of-fit statistics Anderson-Darling and Cramer-von Mises tests have been used, SDWED model proposals the best fitting (see Figure 8 and Figure 9):

\section{Conclusion}

In this paper, Size-biased Double Weighted Exponential Distribution (SDWED) has Table 4. Parameters' estimates and goodness-of-fit statistics.

\begin{tabular}{cccccccccc}
\hline Distributions & $\hat{\theta}$ & $\hat{k}$ & $\hat{\xi}$ & $\hat{\alpha}$ & $\hat{c}$ & $\hat{\lambda}$ & $\hat{\beta}$ & $A_{0}^{2}$ & $W_{0}^{2}$ \\
\hline Size-biased Rayleigh & - & - & - & - & - & - & 46.764 & 0.708 & 0.134 \\
Size-biased Maxwell & - & - & - & 40.50 & - & & - & 1.693 & 0.278 \\
$\begin{array}{c}\text { Weighted Weibull } \\
\quad \text { (size-biased) }\end{array}$ & 0.8151 & 0.604 & 4.759 & - & & & - & 0.1909 & 0.0332 \\
$\begin{array}{c}\text { Size-Biased Double } \\
\text { weighted exponential } \\
\text { distribution(SDWED) }\end{array}$ & & & & & & & & & \\
\hline
\end{tabular}




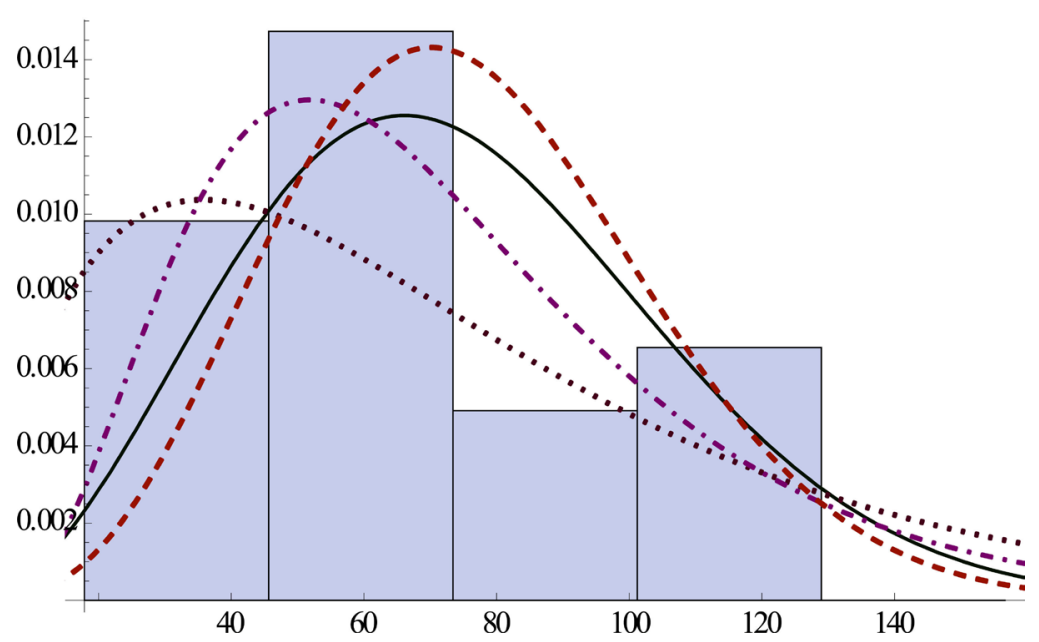

Figure 8. Size-Biased Double Weighted Exponential (Dotted Line), weighted Weibull (Solid Line), Rayleigh (Dashes Line) and Maxwell (dotted dashed Line) on the Histogram.

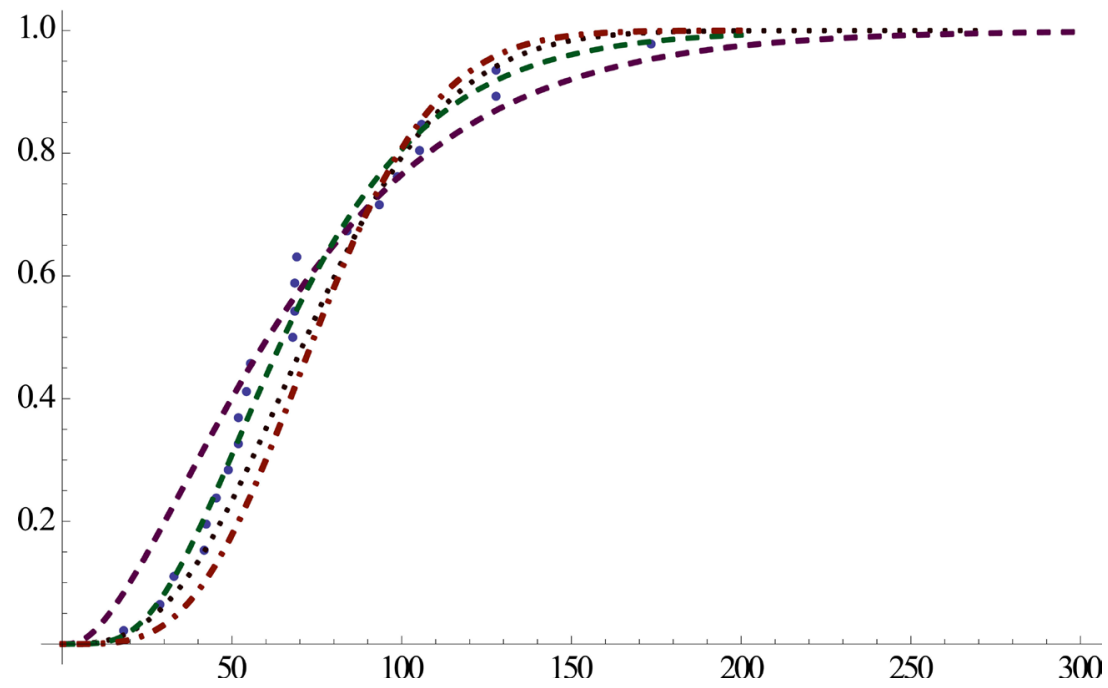

Figure 9. SWWD (dotted Line), SDWED (dott dashed), Maxwell (Solid Line), Rayleigh (Dashed Line), Density Estimates-cdf Estimates and Empirical cdf.

been introduced. The pdf of the SDWED has been studied as well as different reliability measures such as survival function, failure rate function or hazard function. The moments, mode, the coeff. of skewness and the coeff. of kurtosis of SDWED have been derived. For estimating the parameters of SDWED, MLE method has been used. The SDWED has been fitted to Ball Bearing data set. SDWED suggested a good fit of the data as comparing to other distributions.

\section{References}

[1] Fisher, R. (1934) The Effect of Methods of Ascertainment. Annals of Human Genetics, 6, 13-25. http://dx.doi.org/10.1111/j.1469-1809.1934.tb02105.x

[2] Cox, D.R. (1962) Renewal Theory, Metrheun's Monograph. Barnes and Noble, Inc., New York 
[3] Rao, C.R. (1965) ON Discrete Distributions Arising out of Methods of Ascertainment. Sankhya; The Ind.J. Statist, Series A, 27, 311-324.

[4] Cox, D.R. (1968) Some Sampling Problems in Technology. In: Johnson, N.L. and Smith, H., Eds., New Developments in Survey Sampling, John Wiley \& Sons, New York, 506-527.

[5] Zelen, M. (1974) Problems in Cell Kinetics and Early Detection of Disease. Reliability and Biometry, SIAM, Philadelpha, 701-726.

[6] Warren, W.G. (1975) Statistical Distribution of Forestry and Forest Products Research. A Modern Course on Statistical Distributions in Scientific Work. NATO Advanced Study Institutes Series, 17, 369-384. http://dx.doi.org/10.1007/978-94-010-1845-6 27

[7] Rao, C.R. and Patil, G.P. (1977-78) The Weighted Distributions: A Survey of Their Applications. Applications of Statistics, 383-405.

[8] Patil, G.P. and Rao, C.R. (1978) Weighted Distributions and Size-Biased Sampling with Applications to Wildlife Populations and Human Families. Biometrics, 34, 179-189. http://dx.doi.org/10.2307/2530008

[9] Gupta, R.C. and Keating, J.P. (1986) Relations for Reliability Measures under Length Biased Sampling. Scandinavian Journal of Statistics, 13, 49-56.

[10] Arnold, B.C. and Nagaraja, H. (1991) On Some Properties of Bivariate Weighted Distributions. Communications in Statistics-Theory and Methods, 20, 1853-1860. http://dx.doi.org/10.1080/03610929108830602

[11] Jain, K. and Nanda, A.K. (1995) On Multivariate Weighted Distributions. Communications in Statistics-Theory and Methods, 24, 2517-2539. http://dx.doi.org/10.1080/03610929508831631

[12] Patil, G.P. and Ord, J.K. (1976) On Size-Biased Sampling and Related Form-Invariant Weighted Distributions. Industrial Journal of Statistics, 38, 48-61.

[13] Patil, G.P. (1981) Studies in Statistical Ecology Involving Weighted Distributions. Proceedings of the Indian Statistical Institute, Calcutta, 16 December 1981, 478-503.

[14] Mahfoud, M. and Patil, G.P. (1982) On Weighted Distributions. Statistics and Probability, New York, 479-492.

[15] Kochar, S.C. and Gupta, R.P. (1987) Some Results on Weighted Distributions for Positive value Random Variables. Probability in the Engineering and Information Sciences, 1, 417423. http://dx.doi.org/10.1017/S0269964800000498

[16] Oluyede, B.O. (2000) On Some Length Biased Inequalities for Reliability Measures. Journal of Inequalities and Application, 5, 447-466. http://dx.doi.org/10.1155/s1025583400000254

[17] Navarro, J., Aguila, Y.D. and Ruiz, J. (2001) Characterizations through Reliability Measures from Weighted Distributions. Statistical Papers, 42, 395-402. http://dx.doi.org/10.1007/s003620100066

[18] Gove, J.H. (2003) Estimation and Application of Size-Biased Distribution in Forestry. In: Modeling Forest Systems, Northeastern Research Station, USA, 201-211. http://dx.doi.org/10.1079/9780851996936.0201

[19] Sunoj, S.M. and Maya, S.S. (2006) Some Properties of Weighted Distributions in the Context of Repairable Systems. Communications in Statistics - Theory and Methods, 35, 223228. http://dx.doi.org/10.1080/03610920500439968

[20] Shen, Y., Ning, J. and Qin, J. (2009) Analyzing Length-Biased Data with Semi Parametric Transformation and Accelerated Failure Time Models. Journal of the American Statistical Association, 104, 1192-1202. http://dx.doi.org/10.1198/jasa.2009.tm08614

[21] Hussain, A. and Ahmed, P.B. (2009) Misclassification in Size-Biased Modified Power Series 
Distribution and Its Applications. Journal of KSIAM, 13, 55-72.

[22] Mir, K.A. and Ahmad, M. (2009) Size-Biased Distributions and Their Applications. Pakistan Journal of Statistics, 25, 283-294.

[23] Mir, K.A. and Ahmad, M. (2009) On Size-Biased Geeta Distribution. Pakistan Journal of Statistics, 25, 309-316.

[24] Das, K.K. and Roy, T.D. (2011) Applicability of Length Biased Weighted Generalized Rayleigh Distribution. Advances in Applied Science Research, 2, 320-327.

[25] Dara, S.T. (2011) Recent Advances in Moment Distributions and Their Hazard Rates. Ph.D. Dissertation, National College of Business Administration and Economics, Lahore.

[26] Iqbal, Z. and Ahmad, M. (2012) Some Properties of Generalized Log-Pearson Distribution. Proceeding of 12 th International Islamic Countries Conference on Statistical Sciences, Doha, Vol. 23, 285-296.

[27] Hasnain, S.A. (2013) Exponentiated Moment Exponential Distribution. Ph.D. Thesis, National College of Business Administration \& Economics, Lahore.

[28] Iqbal, Z., et al. (2014) Generalized Exponentiated Moment Exponential Distribution. Pakistan Journal of Statistics, 30, 537-554.

[29] Zahida (2014-2015) On the Weighted and Weighted Double Weibull Distributions. Ph.D. Dissertation, National College of Business Administration and Economics, Lahore.

[30] Lawless, J. (1982) Statistical Models and Methods for Lifetime Data. John Wiley \& Sons, New York. for you:

Accepting pre-submission inquiries through Email, Facebook, LinkedIn, Twitter, etc. A wide selection of journals (inclusive of 9 subjects, more than 200 journals)

Providing 24-hour high-quality service

User-friendly online submission system

Fair and swift peer-review system

Efficient typesetting and proofreading procedure

Display of the result of downloads and visits, as well as the number of cited articles

Maximum dissemination of your research work

Submit your manuscript at: http://papersubmission.scirp.org/

Or contact ojs@scirp.org 